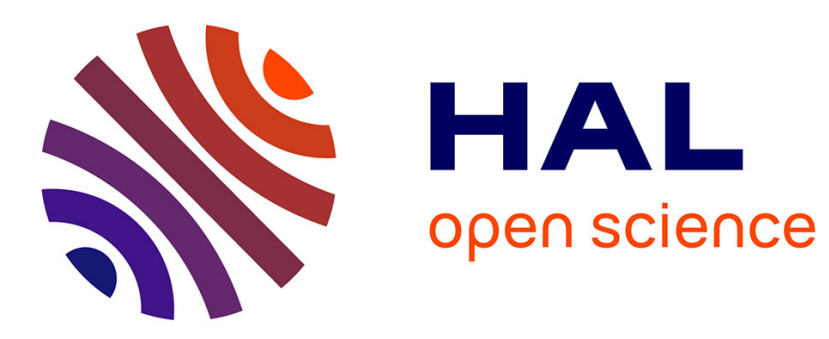

\title{
Multiscale deformation of a liquid surface in interaction with a nanoprobe
}

\author{
René Ledesma-Alonso, Philippe Tordjeman, Dominique Legendre
}

\section{To cite this version:}

René Ledesma-Alonso, Philippe Tordjeman, Dominique Legendre. Multiscale deformation of a liquid surface in interaction with a nanoprobe. Physical Review E : Statistical, Nonlinear, and Soft Matter Physics, 2012, vol. 85, pp. 1-10. 10.1103/PhysRevE.85.061602 . hal-00904859

\section{HAL Id: hal-00904859 \\ https://hal.science/hal-00904859}

Submitted on 15 Nov 2013

HAL is a multi-disciplinary open access archive for the deposit and dissemination of scientific research documents, whether they are published or not. The documents may come from teaching and research institutions in France or abroad, or from public or private research centers.
L'archive ouverte pluridisciplinaire HAL, est destinée au dépôt et à la diffusion de documents scientifiques de niveau recherche, publiés ou non, émanant des établissements d'enseignement et de recherche français ou étrangers, des laboratoires publics ou privés. 


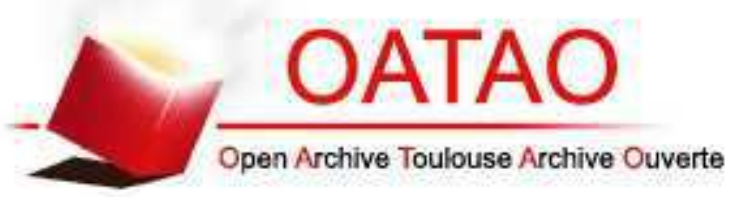

\section{Open Archive TOULOUSE Archive Ouverte (OATAO)}

OATAO is an open access repository that collects the work of Toulouse researchers and makes it freely available over the web where possible.

This is an author-deposited version published in : http://oatao.univ-toulouse.fr/ Eprints ID : 10101

To link to this article : DOI:10.1103/PhysRevE.85.061602

URL : http://dx.doi.org/10.1103/PhysRevE.85.061602

To cite this version : Ledesma-Alonso, René and Tordjeman, Philippe and Legendre, Dominique. Multiscale deformation of a liquid surface in interaction with a nanoprobe. (2012) Physical Review E, vol. 85 (nº). pp. 1-10. ISSN 1539-3755

Any correspondance concerning this service should be sent to the repository administrator: staff-oatao@ listes-diff.inp-toulouse.fr 


\title{
Multiscale deformation of a liquid surface in interaction with a nanoprobe
}

\author{
R. Ledesma-Alonso, P. Tordjeman, and D. Legendre \\ Université de Toulouse, INPT-CNRS, Institut de Mécanique des Fluides de Toulouse (IMFT), 1 Allée du Professeur Camille Soula, \\ 31400 Toulouse, France
}

\begin{abstract}
The interaction between a nanoprobe and a liquid surface is studied. The surface deformation depends on physical and geometric parameters, which are depicted by employing three dimensionless parameters: Bond number $B_{o}$, modified Hamaker number $H_{a}$, and dimensionless separation distance $D^{*}$. The evolution of the deformation is described by a strongly nonlinear partial differential equation, which is solved by means of numerical methods. The dynamic analysis of the liquid profile points out the existence of a critical distance $D_{\min }^{*}$, below which the irreversible wetting process of the nanoprobe happens. For $D^{*} \geqslant D_{\min }^{*}$, the numerical results show the existence of two deformation profiles, one stable and another unstable from the energetic point of view. Different deformation length-scales, characterizing the stable liquid equilibrium interface, define the near- and the far-field deformation zones, where self-similar profiles are found. Finally, our results allow us to provide simple relationships between the parameters, which leads to determine the optimal conditions when performing atomic force microscope measurements over liquids.
\end{abstract}

\section{INTRODUCTION}

The study of fluids and their properties at the nanoscopic scale by means of local probe techniques is still today a big challenge. Involving molecular interaction forces, several apparatuses and techniques have been in constant development for the past four decades, mainly the surface force apparatus (SFA) and the atomic force microscope (AFM). Even though the SFA gives a vertical distance resolution of about $0.1 \mathrm{~nm}$ and a force sensitivity of $10 \mathrm{nN}$ [1], its lateral resolution is limited by the radius of the two approaching cylinders, around $1 \mathrm{~cm}$, on which the technique is based. Nevertheless, its configuration is optimum for studying rheological properties of thin films [2]. On the other hand, the AFM provides a similar vertical resolution and an augmented force sensitivity of $10 \mathrm{pN}$ [3], whereas its lateral resolution is given by the size of the tip radius, commonly between $10-20 \mathrm{~nm}$. Hence, AFM techniques allow the acquisition of detailed topographies and the visualization of different material phases, among other properties involving molecular interactions [4-7]. However, in spite of its advantages and possible applications, the characterization of liquid surfaces by means of AFM methods has been delayed due to the difficulties induced by the interface deformation, the jumping capillarity phenomenon, and the inherent probe wetting.

The geometry of an ordinary SFA experiment allows the employment of the Derjaguin approximation, which has been proven to be appropriate in most cases. For common AFM nanoprobes, it is not applicable because the tip radius has a smaller or similar size compared to the gap between the deformed liquid surface and the tip [8]. Previously, the basis of a theoretical model to compute the liquid surface deformation were developed [9], in which no geometric hypothesis was made, providing a valid analysis at any length-scale. Taking into account the molecular attractive interaction, represented by a modified Hamaker number, and neglecting the effect of gravity, depicted by the Bond number, the height of the deformation profile was estimated. Good agreement between AFM experiments and the deformation force computed with the model were achieved, validating the introduced methodology.
Herein, we present an extensive analysis, which deepens into the parametric study and its consequences over the dynamic evolution of the interface profile, at different length-scales. The results show that the deformation extends beyond the tip radius, and as far as the capillary length, which for the case of local probes (tip radius $\sim 10^{-8} \mathrm{~m}$ ) is several orders of magnitude greater (capillary length $\sim 10^{-3} \mathrm{~m}$ ). Different deformation zones are portrayed and delimited by different characteristic length-scales: from the origin of an axisymmetric reference system up to a length-scale given by the Hamaker interaction force, a near-field is found; from a transition length-scale up to the capillary length, a far-field spans; and between the two previous zones, a transition or linking zone is located, which extension depends on the combined effect of the attractive interaction and capillarity parameters. Keeping in mind the different length-scales, we find that the deformation profile shows a self-similar behavior.

In addition, a particular relationship between the apex deformation and its curvature is found. The resulting fit serves to calculate the minimum distance at which the probe can approach the liquid without being wetted, as well as its corresponding maximum deformation. These quantities result to be functions of the dimensionless parameters, and can be employed to determine the optimal experimental conditions when AFM measurements are performed over liquid surfaces.

\section{INTERFACE DEFORMATION}

\section{A. Model}

Considering that the London-van der Waals (London$\mathrm{vdW}$ ) interaction force between molecules acts over a relatively short distance, only the rounded extreme of the tip generates a reaction at the interface [9]. Consequently, in order to understand the nature of the noncontact interaction between a local probe and a deformable material surface, we model the tip as a sphere of radius $R$. Within a cylindrical coordinate system, a perfectly rigid sphere is placed at a fixed position over a semi-infinite liquid body, which is deformed like the one shown in Fig. 1, the gap between them being filled with air. 


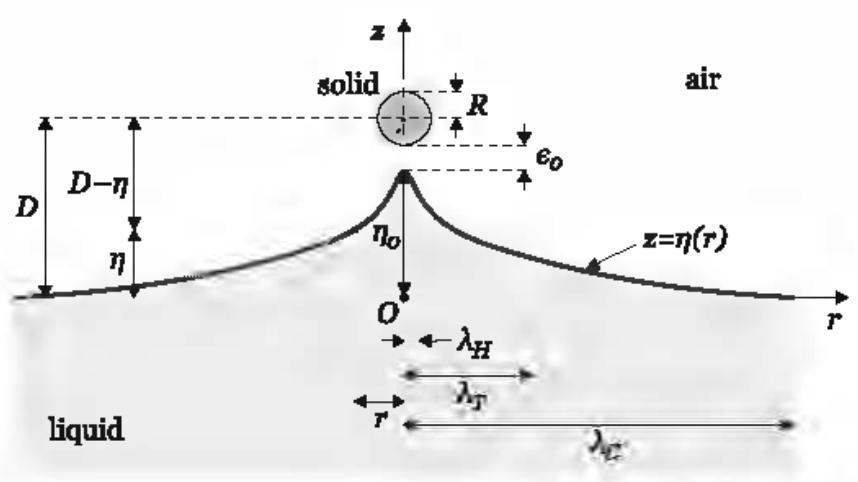

FIG. 1. Scheme of the liquid surface deformation in interaction with a probe. Parameters defined in the text.

The interface is initially flat and located at $z=0$. Based on the Hamaker theory [10], the pressure field (or attractive potential) $\Pi$ exerted by the sphere at a distance $D$ over the bulk liquid is

$$
\Pi=\frac{4 H R^{3}}{3 \pi} \frac{1}{\left[(D-z)^{2}+r^{2}-R^{2}\right]^{3}},
$$

where $H$ is the Hamaker constant of the probe/air/liquid system, while $z$ and $r$ are the vertical and the radial coordinates of any point in the liquid, os show in Fig. 1. Equation (1) is obtained from integrating the London-vdW interaction potential between the sphere atoms and a differential volume at a given distance from the sphere surface. As shown in Fig. 2, the radial extent of the pressure field is smaller than the sphere radius. As well, considering the dilated vertical axis, which allows the visualization of the corresponding equilibrium liquid surface, the depth barely

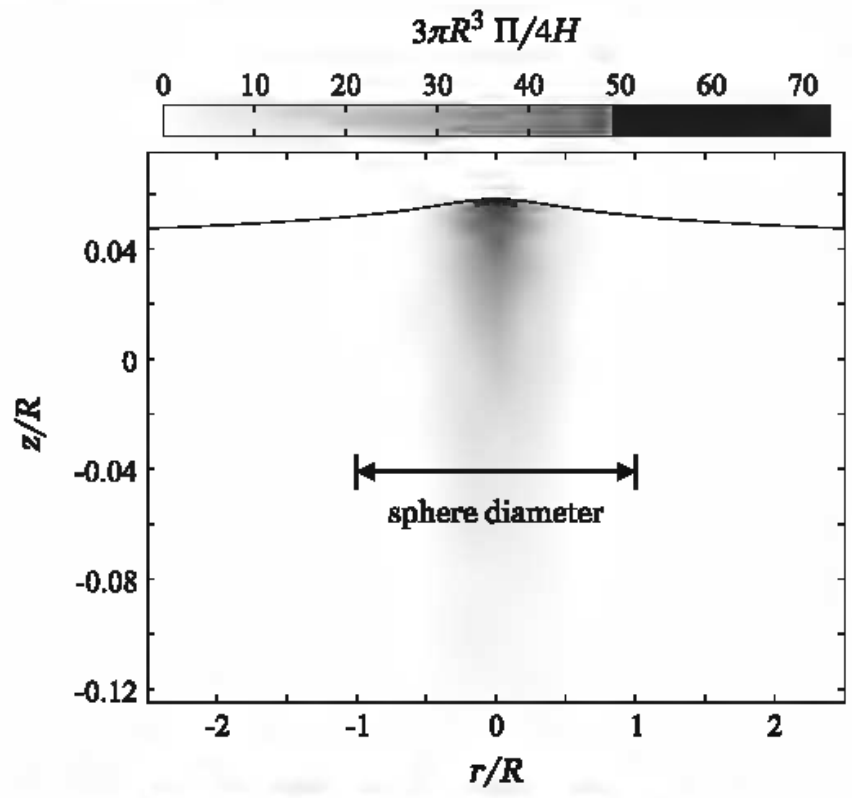

FIG. 2. Presgare field over the liquid body generated by the Interaction with the spherical probe, obtained from $\mathrm{Bq}$. (1), and the corresponding interface deformed position for $H=4 \times 10^{-20} \mathrm{Nm}$ and $R=10^{-0} \mathrm{~m}$. The vertical axis is magnified to facilitate the interface visual recognition. reaches 0.1 times the sphere radius. In brief, the interaction effect is restricted to a very small zone over the liquid phase.

In turn, this attractive potential tends to deform the interface, which provokes a capillary pressure difference and a hydrostatic pressure in order to balance the system, as well as a dynamic contribution entailed by the yiscous dissipation of the system. As a first approach, we have disregarded the inertial effect with respect to the viscous contribution. Thence, the generalized Young-Laplace's equation without mass transfer, which describes the interface behavior, is expressed as

$$
\Pi-\Delta \rho g \eta+\underline{n} \cdot \Sigma \cdot \underline{n}=2 \kappa \gamma,
$$

where $\Delta \rho$ is the density difference between the liquid phase and the air, $g$ is the gravitational acceleration, $\underline{n} \cdot \Sigma \cdot \underline{n}$ is the normal viscous stress difference across the interface characterized by the unit normal vector $\underline{n}_{2} \kappa$ is the local interface mean curvature, $\gamma$ is the surface tension, and $\eta$ is the local interface position, which within an axisymmetric model is given as a function of the radial position $r$ and the time $t$. Within this portrayal, inertia was disregarded with respect to viscous dissipation because, at the AFM scale, the Reynolds number is of the order of $\operatorname{Re} \in\left[10^{-9}, 10^{0}\right]$.

Additionally, considering a general case for which large deformations are envisaged, the expression of the curvature in cylindrical coordinates is given by

$$
2 x=-\frac{1}{r} \frac{\partial}{\partial r}\left[r \frac{\partial \eta}{\partial r}\left\{\left(\frac{\partial \eta}{\partial r}\right)^{2}+1\right\}^{-1}\right] .
$$

As well, in the more simplistic case, the viscous stresses at the air-side are negligible with respect to those at the liquid-side. Hence, for an incompressible single-directional viscous flow, which moves mainly in the $z$ direction, and considering that in cylindrical coordinates $\underline{n}$ is approximated by $n_{r} \approx-\partial \eta / \partial r$ and $n_{z} \approx 1$, we have

$$
\underline{n} \cdot \Sigma \cdot \underline{n} \approx-\mu \frac{\partial}{\partial t}\left(\frac{\partial \eta}{\partial r}\right)^{2}
$$

where $\mu$ represents the viscosity of the liquid. We can introduce the definitions of the capillary length, $\lambda_{C}=\sqrt{\gamma /(\Delta \rho g)}$, and the relaxation time, $\tau=(R \mu) / \gamma$. of the system. As well, considering $R$ as the characteristic length-scale and $\tau$ as the characteristic time-scale of the system, we find the dimensionless variables: $D^{*}=D / R$ the distance from the center of the sphere to the originally undeformed interface, $r^{*}=r / R$ and $z^{*}=z / R$ the horizontal and vertical coordinates, $\eta^{*}=\eta / R$ and $\kappa^{*}=\kappa R$ the deformation and curvature, $\lambda_{C}^{*}=\lambda_{C} / R$ the dimensionless capillary length, and $t^{*}=t / \tau$ the dimensionless time. Thus, matching Eqs. (1) - (4), the nonlinear partial differential equation that describes the instantaneous position of the interface is written as the following dimensionless expression:

$$
\begin{aligned}
& -\frac{1}{r^{*}} \frac{\partial}{\partial r^{*}}\left[\frac{r^{*} \frac{\partial \eta^{*}}{\partial r^{*}}}{\left(\frac{\partial \eta^{*}}{\partial r^{*}}\right)^{2}+1}\right]+B_{o} \eta^{*}-\frac{H_{a}}{\left[\left(D^{*}-\eta^{*}\right)^{2}+r^{* 2}-1\right]^{3}} \\
& \quad=-\frac{\partial}{\partial t^{*}}\left(\frac{\partial \eta^{*}}{\partial r^{*}}\right)^{2}
\end{aligned}
$$


in which we find two dimensionless parameters: the Bond number $B_{o}=\left(R / \lambda_{C}\right)^{2}$ and the modified Hamaker number $H_{a}=4 H /\left(3 \pi \gamma R^{2}\right)$.

This dimensionless analysis gives rise to an equation that is valid for a system of any length-scale. In addition, as it is clearly observed from the parameter definitions, $H_{a}$ and $B_{o}$ are coupled by $R$. Hence, their values are restricted according to the product of physical properties given by:

$$
H_{a} B_{o}=\frac{4}{3 \pi}\left(\frac{H \Delta \rho g}{\gamma^{2}}\right) \text {. }
$$

Considering real probe/air/liquid systems [1,11-13], for which $H \in\left[10^{-21}, 10^{-19}\right] \mathrm{J}, \quad \gamma \in\left[10^{-2}, 10^{-1}\right] \mathrm{N} / \mathrm{m}, \quad \Delta \rho \in$ $\left[10^{2}, 10^{4}\right] \mathrm{kg} / \mathrm{m}^{3}$, and $\mu \in\left[10^{-3}, 10^{0}\right] \mathrm{Pa} \mathrm{s}$, and common AFM probes, with $R \in\left[10^{-8}, 10^{-7}\right]$, the range of the dimensionless parameters remains within $H_{a} \in\left[10^{-8}, 10^{-1}\right]$ and $B_{o} \in$ $\left[10^{-11}, 10^{-8}\right]$

For simplicity, the deformation, and its first and second derivatives with respect to $r^{*}$ are written as $\eta_{0}^{*}$, $\left[\eta^{*}\right]_{0}^{\prime}$, and $\left[\eta^{*}\right]_{0}^{\prime \prime}$, respectively, when evaluated at $r^{*}=0$, and as $\eta_{C}^{*},\left[\eta^{*}\right]_{C}^{\prime}$, and $\left[\eta^{*}\right]_{C}^{\prime \prime}$, respectively, when evaluated at $r^{*}=\lambda_{C}^{*}$.

At $r^{*}=0$, a symmetry boundary condition, $\left[\eta^{*}\right]_{0}^{\prime}=0$, should be considered. Meanwhile, far from the axis at $\lambda_{C}^{*} \gg 0$, where the interaction potential, which decays as $\left(r^{*}\right)^{-6}$, is negligible $(\Pi \approx 0)$, the boundary condition comes from the quasistatic asymptotic solution of Eq. (5), also considering that in this outlying region the surface is nearly flat $\left(\left[\eta^{*}\right]^{\prime} \ll 1\right)$. The exact solution, which leads to the corresponding faraway boundary condition, is

$$
\eta^{*}=G K_{0}\left(\sqrt{B_{o}} r^{*}\right),
$$

with the coefficient $G=\eta_{C}^{*} / K_{0}(1)$, and $K_{0}$ being a zero-order modified Bessel function of the second kind. Hence, the boundary condition at $r^{*}=\lambda_{C}^{*}$ is given by

$$
\left[\eta^{*}\right]_{C}^{\prime}+\sqrt{B_{o}} \frac{K_{1}(1)}{K_{0}(1)} \eta_{C}^{*}=0,
$$

where $K_{1}$ is a first-order modified Bessel function of the second kind.

We suppose that the interface is undeformed and completely flat before the dynamic process starts. Hence, the initial condition at $t=0$, is given by $\eta^{*}=\left[\eta^{*}\right]^{\prime}=\kappa^{*}=0$ for all $r^{*} \geqslant 0$, which is equivalent to say that the sphere is suddenly set at $t=0$.

After a time interval close to $\tau$, a steady-state profile is expected to be obtained. Considering real probe/air/liquid systems, we find $\tau \in\left[10^{-6}, 10^{-10}\right] \mathrm{s}$ for AFM situations. Because of the relatively small magnitude of this characteristic time-scale compared to the common laboratory measured time-scales, as a second approach, we consider that the steady state is reached instantaneously. At equilibrium, liquid and interface are motionless, viscous stresses do not appear and the temporal derivative in Eq. (5) is omitted. Thus, Eq. (5) is transformed into a dimensionless nonlinear ordinary differential equation and, as expected, the equilibrium profile is obtained when the interaction pressure field is totally compensated by the gravity and the capillary contributions.

A posteriori, it was verified that $\left(\left[\eta^{*}\right]^{\prime}\right)^{2} \ll 1$, which supports the employment of the small interface displacements hypothesis in our theoretical analysis. Taking this into account, the dimensionless mean curvature $\kappa^{*}$ is decomposed in two principal curvatures

$$
\begin{aligned}
2 \kappa^{*} & =\kappa_{m}^{*}+\kappa_{a}^{*} \\
\kappa_{m}^{*} & =-\frac{d^{2} \eta^{*}}{d r^{* 2}} \quad \kappa_{a}^{*}=-\frac{1}{r^{*}} \frac{d \eta^{*}}{d r^{*}},
\end{aligned}
$$

where $\kappa_{m}^{*}$ is the dimensionless meridional curvature-the axisymmetric curvature of the interface that determines the behavior of the profile at any axial plane-while $\kappa_{a}^{*}$ is the dimensionless azimuthal curvature - the curvature projection of the circle describing isodeformation contour lines in the direction normal to the interface.

\section{B. Numerical method}

Due to the impossibility of solving analytically the strongly nonlinear Eq. (5), a numerical method was implemented. Firstly, taking care of the singularity present at $r^{*}=0$, we consider the Taylor expansion

$$
\frac{1}{r^{*}} \frac{d \eta^{*}}{d r^{*}} \approx\left[\eta^{*}\right]_{0}^{\prime \prime}+O\left(r^{*}\right)
$$

Additionally, employing the finite difference method to discretize the time derivative, we can write the dynamic term as

$$
\frac{\partial}{\partial t^{*}}\left(\frac{\partial \eta^{*}}{\partial r^{*}}\right)^{2}=\frac{1}{\Delta t^{*}}\left\{\left.\left(\frac{\partial \eta^{*}}{\partial r^{*}}\right)^{2}\right|^{n}-\left.\left(\frac{\partial \eta^{*}}{\partial r^{*}}\right)^{2}\right|^{n-1}\right\},
$$

where $n$ and $n-1$ indicate two consecutive time-steps, and $\Delta t^{*}$ is the dimensionless time-step of the simulation.

Now, writing Eq. (5) as a system of nonlinear ordinary differential equations, we have to solve

$$
\begin{aligned}
& \left.\frac{d \eta^{*}}{d r^{*}}\right|^{n}=u \\
& \left.\frac{d u}{d r^{*}}\right|^{n}= \begin{cases}-\frac{u\left[u^{2}+1\right]}{r^{*}}+\left[B_{o} \eta^{*}-\frac{H_{a}}{\left(\left[D^{*}-\eta^{*}\right]^{2}+r^{* 2}-1\right)^{3}}+\frac{u^{2}-A}{\Delta t^{*}}\right]\left[u^{2}+1\right]^{3 / 2}, & \forall r^{*}>0 \\
{\left[B_{o} \eta^{*}-\frac{H_{a}}{\left(\left[D^{*}-\eta^{*}\right]^{2}+r^{* 2}-1\right)^{3}}+\frac{u^{2}-A}{\Delta t^{*}}\right] \frac{\left[u^{2}+1\right]^{3 / 2}}{u^{2}+2},} & r^{*}=0,\end{cases}
\end{aligned}
$$


where $u$ is the first spatial derivative of the liquid surface position, $\eta^{*}=\left.\eta^{*}\right|^{n}$ for simplicity, and $A=\left.\left(d \eta^{*} / d r^{*}\right)^{2}\right|^{n-1}$ is a numerical method parameter. The problem represented by Eqs. (12) must be solved to obtain the profile of the interface at any time $n$, with the knowledge of the profile at the previous instant $n-1$. This system is a two-point boundary value problem, which is worked out using a MATLAB routine including the function bvp4c.m, which employs the so-called Simpson's method [14]. Because of the smooth shape of the composing functions of the system expressed in Eqs. (12), the solution is easily found with the proposed method.

The boundary and initial conditions are

$$
\begin{aligned}
& r^{*}=0 \Rightarrow u=0 \\
& r^{*}=\lambda_{C}^{*} \Rightarrow u=-\sqrt{B_{o}} \frac{K_{1}(1)}{K_{0}(1)} \eta_{C}^{*} \\
& \left.\begin{array}{c}
t=0 \\
0 \leqslant r^{*} \leqslant \lambda_{C}^{*}
\end{array}\right\} \Rightarrow\left\{\begin{array}{l}
\eta^{*}=\eta_{i}^{*}\left(1-\frac{r^{*}}{\lambda_{c}^{*}}\right) \\
u=-\frac{\eta_{i}^{*}}{\lambda_{C}^{*}},
\end{array}\right.
\end{aligned}
$$

where $\eta_{i}^{*}$ is the initial guess of the interface apex position. Due to the nonlinearity of the system, two different shapes of the interface profile can be found as simultaneous solutions of Eq. (5), depending on the value of $\eta_{i}^{*}$ (this point is discussed in Sec. III C). For the dynamic calculation of the profile evolution, the surface is initially placed at $\eta_{i}^{*}=0$. For steady-state calculation, the dynamic system is transformed into the equivalent system of equations, which describes the static case. The previous and the current instants are then nullified, and we take $A=u^{2}$ and suppress the superindex $n$. For all the situations, dynamic or static, interface profiles are calculated with a relative tolerance of $10^{-4}$.

\section{LIQUID SURFACE DEFORMATION}

\section{A. Transient state}

From the results presented in the literature [9], for a given combination of the parameters $H_{a}$ and $B_{o}$, the evolution of the interface profile depends completely on the relative value of $D^{*}$. If $D^{*}$ is greater than a threshold value, $D_{\min }^{*}$, a bumplike equilibrium profile is attained. On the other hand, if $D^{*}$ is smaller than $D_{\min }^{*}$, the profile deformation grows until the liquid touches the sphere, developing the so-called jumpto-contact processes and the formation of a liquid capillary bridge [15].

A typical interface profile evolving and reaching an equilibrium state is shown in Fig. 3(a). As well, the different evolution paths that the interface apex can follow, depending on $D^{*}$, are shown in Fig. 3(b). For all the considered values of $D^{*}$, the instantaneous application of the attractive force at $t=0$ directly results into an abrupt acceleration of the interface. In turn, surface tension generates a restoring force, which acts to oppose the interface deformation. As a consequence, the driving force in the next instant results from the balance between attractive and surface tension forces, both increasing along with the deformation. At the initial stage, the interface speed decreases monotonically due to the viscous dissipation within the liquid phase, which tends to damp the bulk liquid motion. If $D^{*}>D_{\min }^{*}$, the attractive force is sufficiently small to be controlled by the surface tension, and

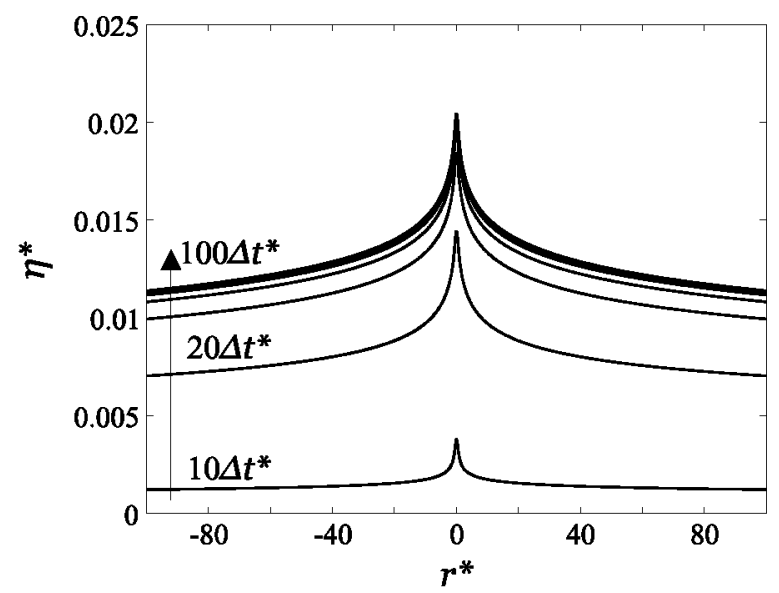

(a)

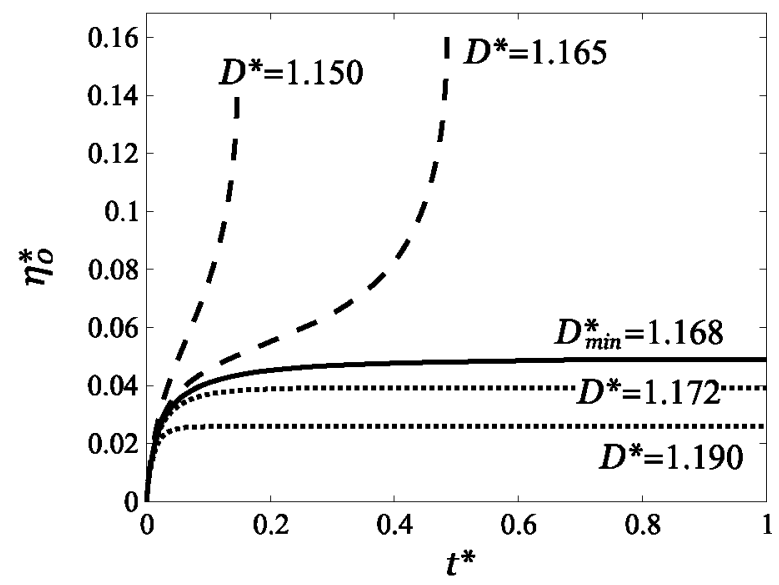

(b)

FIG. 3. Interface dynamic evolution obtained from solving Eqs. (12) for $H_{a}=10^{-3}, B_{o}=10^{-10}$, and $\Delta t^{*}=10^{-3}$. (a) Instantaneous dimensionless profiles for $D^{*}=1.2>D_{\min }^{*}$, corresponding to increments of $10 \Delta t^{*}$ (from bottom to top). (b) Time-dependent dimensionless apex position for: [- $] D^{*}<D_{\min }^{*}$, [-] $D^{*}=D_{\min }^{*}$, and $[\cdots] D^{*}>D_{\min }^{*}$.

the interface motion slows down until a steady-state profile is achieved, as represented by the dotted curves in Fig. 3(b). The solid curve indicates the critical dynamic evolution, for which $D^{*}=D_{\min }^{*}$ and the profile converges slowly toward an equilibrium state, attained near $t^{*} \approx 1$. In contrast, when $D^{*}<D_{\min }^{*}$, the attractive force is large enough to go beyond the tension force, and the motion grows without a regulating mechanism, leading to the sphere-liquid contact. The profile begins normally its deformation and the speed decreases until it reaches a temporary shrinkage, followed by a rise of the speed, the profile divergence, and the subsequent wetting process. Also in Fig. 3(b), the dashed curves describe this behavior, characterized by the so-called jump-to-contact process.

\section{B. Steady-state equilibrium profile}

The interface profile converges toward a final steady-state solution only if $D^{*} \geqslant D_{\min }^{*}$. An example of the typical equilibrium deformation profiles are shown in Fig. 4, for fixed values of $H_{a}$ and $B_{o}$, and an initially flat surface placed at $\eta_{i}^{*}=0$, but for different values of $D^{*}$. The corresponding curvatures, $\kappa_{m}^{*}$ and $\kappa^{*}$ [see Eq. (9)], are displayed in Fig. 5. As it 
(a)

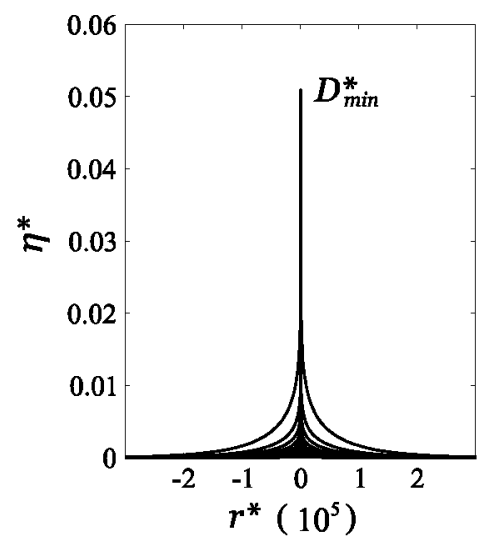

(b)

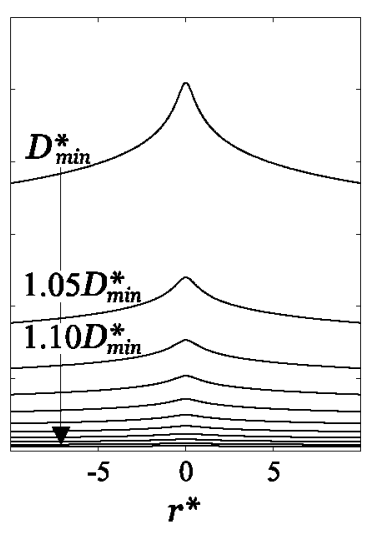

FIG. 4. (a) Steady-state dimensionless equilibrium profiles and (b) zoom of the central region obtained from solving the steady-state Eq. (5), for $H_{a}=10^{-3}, B_{o}=10^{-10}$, an initial guess with $\eta_{i}^{*}=0$, and $D^{*}$ with increments of $0.05 D_{\text {min }}^{*}$ (from top to bottom). In this case, it was found that $D_{\min }^{*}=1.168$.

is clearly depicted in the deformation profile shown in Fig. 4(a), the sphere pulls the interface towards itself only at a very reduced zone near the symmetry axis, as shown in Fig. $4(\mathrm{~b})$, which is a central region zoom. From $r^{*} \approx 10^{2}-10^{3}$ and beyond, the asymptotic solution expressed in Eq. (7) perfectly describes the declining capillary behavior of the deformation. In addition, the meridional and mean curvatures are shown in Figs. 5(a) and 5(b), respectively. There is a passage from a negative to a positive value of $\kappa_{m}^{*}$, near $r^{*}=0.37$, which indicates a change in the sense of the surface tension force in the axial plane. On the contrary, $\kappa^{*}$ remains always within positive values, showing a narrow logisticlike behavior, barely covering a projected radius. In short, from the curves in Figs. 4 and 5 , reducing $D^{*}$ provokes an increment in the magnitude of the attractive force, leading to greater deformation and curvature states, as well as a slight variation of the radial extension of both.

\section{Bifurcation diagrams}

In fact, for each combination of the parameters $H_{a}, B_{o}$, and $D^{*}$, two solutions for the deformation profile arise from the initial value chosen for $\eta_{i}^{*}$. By taking $D^{*}$ and the two corresponding values of $\eta_{0}^{*}$ as coordinate pairs, we obtain a bifurcation curve. Examples are shown in Fig. 6(a) for a fixed value $B_{o}=10^{-10}$. When $D^{*}=D_{\min }^{*}$, there is only one solution profile, corresponding to the maximum apex deformation $\eta_{\max }^{*}$ and the minimum gap $\epsilon_{\min }^{*}$ between the liquid surface and the sphere. There is no solution for the deformation profile further to the left than this critical point $\left(D_{\min }^{*}, \eta_{\max }^{*}\right)$, from which the two branches emerge.

As observed from Fig. 6(a), the curve showing the highest apex deformation is thus an unstable branch from the energetic point of view. As well, the curve reporting the lowest apex position, associated to the minimum deformation energy, is a stable branch and thus describes the only possible interface profile. For the stable apex position, the dependency of $\eta_{0}^{*}$ on $D^{*}$ seems to take the form of a rectangular hyperbola:

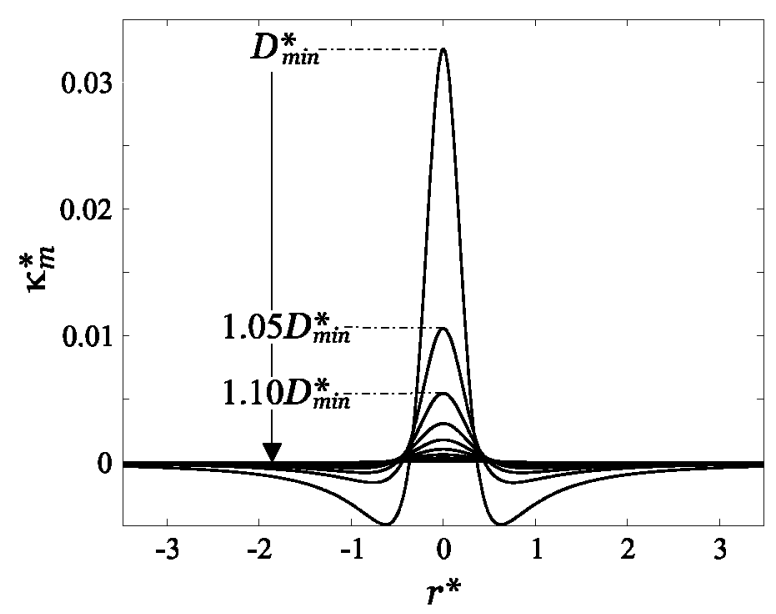

(a)

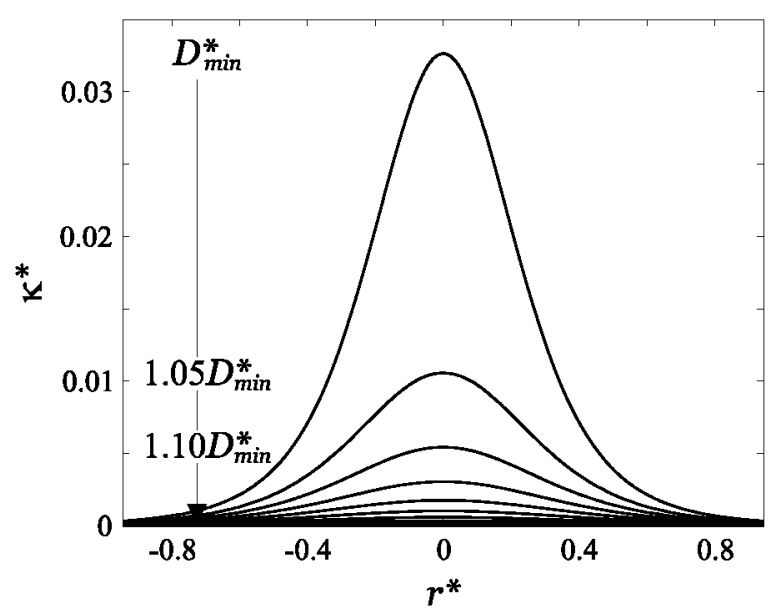

(b)

FIG. 5. Steady-state dimensionless equilibrium (a) meridional curvatures and (b) local mean curvatures obtained from solving the steady-state Eq. (5), with the same parameter values and variables as in Fig. 4.

the shorter the gap between the probe and the undeformed surface, the larger the deformation becomes. In Figs. 6(b) and $6(\mathrm{c})$, the derivative of $\eta_{0}^{*}$ with respect to $D^{*}$ and the curvature of the stable branch are shown, respectively. In both cases, $D_{\min }^{*}$ marks the location of a vertical asymptote, at which the stable branches are completely halted.

An increase of $H_{a}$ generates a displacement of the bifurcation curve to larger values of $D^{*}$, as well as a proportional scaling. Conversely, an increase of $\boldsymbol{B}_{o}$ provokes a very small decrease of $D^{*}$, which is negligible in comparison with those provoked by $H_{a}$. In general, the impact of $H_{a}$ is significant, while the effects of a change in $B_{o}$ are negligible within the range of common liquids, as it is summarized in Fig. 7. Increasing $H_{a}$ provokes an enlargement of $\eta_{\max }^{*}$ and $D_{\min }^{*}$; while, if $B_{o}$ increases, both $\eta_{\max }^{*}$ and $D_{\min }^{*}$ show slightly smaller values.

\section{Geometric relations}

Noncontact AFM experiments require to control the tip/liquid distance, which is a function of their physical properties. This can be done by considering the geometric relations that are presented below. When analyzing the apex 


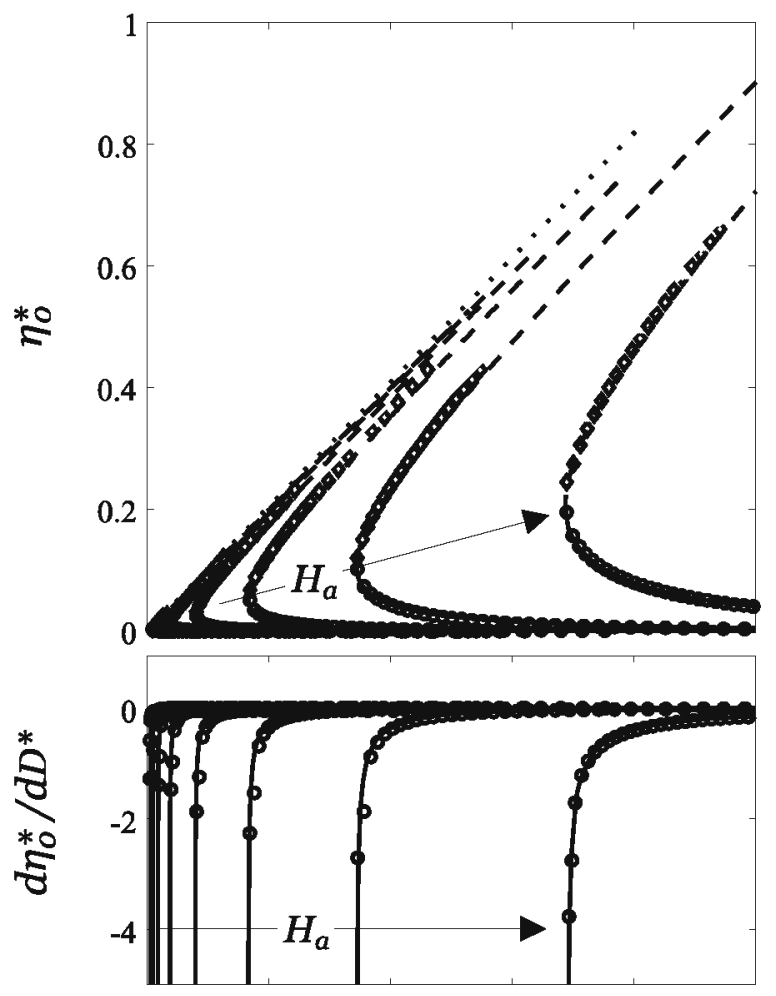

(a)

(b)

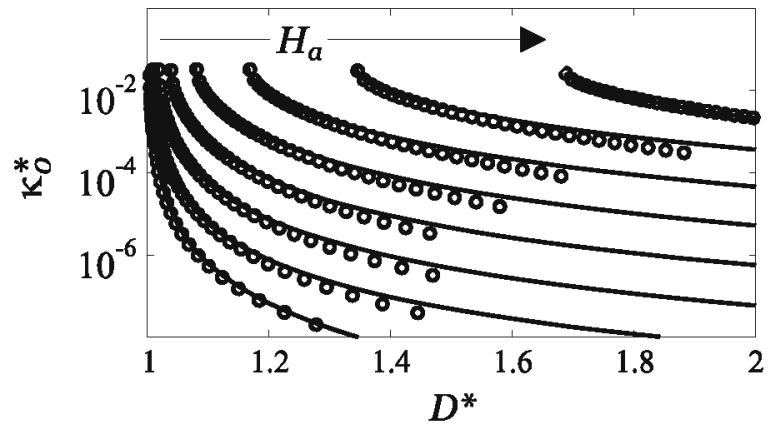

(c)

FIG. 6. Bifurcation diagrams: (a) apex deformation of the interface, (b) its derivative with respect to the separation distance, and (c) its mean curvature, as functions of $D^{*}$, for $H_{a} \in\left[10^{-8}, 10^{-1}\right]$ and $B_{o}=10^{-10}$. Numerical solution of the steady-state Eq. (5) for [o] the stable and $[\diamond]$ the unstable branches, and the analytical solution for [-] the stable and [- -] the unstable branches given by Eqs. (14) and (15). In (a) the position of the sphere surface $[\cdot]$ is shown.

behavior for a given combination of $H_{a}$ and $B_{o}$, it is found that $\kappa_{0}^{*}$ grows along with $\eta_{0}^{*}$, which in turn becomes larger when $D^{*}$ decreases, as observed in Fig. 8. The curvature $\kappa_{0}^{*}$ shows a remarkable simple dependency on $\eta_{0}^{*}$, given by

$$
\kappa_{0}^{*}=C\left(\eta_{0}^{*}\right)^{3 / 2}
$$

in which the prefactor $C \approx 0.4\left(B_{o}^{0.06} / H_{a}^{0.5}\right)$ is obtained from fitting the solutions of $\mathrm{Eq}$. (5) for the range of parameters considered. From Fig. 8, a horizontal logarithmic displacement is produced when modifying the dimensionless numbers: significantly toward larger values of $\eta_{0}^{*}$ when $H_{a}$ increases; barely noticeable to smaller values of $\eta_{0}^{*}$ when $B_{o}$ grows. While $\eta_{0}^{*}$ moves towards $\eta_{\max }^{*}, \kappa_{0}^{*}$ reaches its maximum value

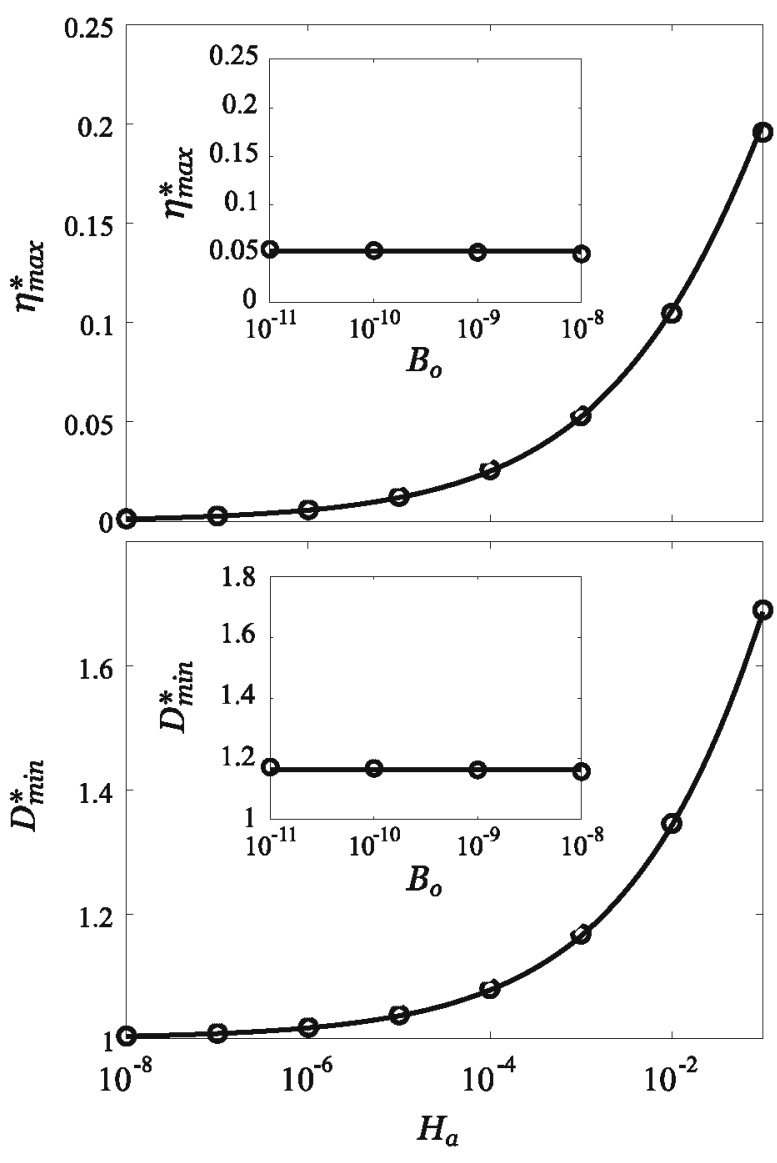

(a)

(b)

FIG. 7. (a) Maximum apex deformation and (b) minimal dimensionless distance as functions of $H_{a}$ for a fixed $B_{o}=10^{-10}$, and (insets) as functions of $B_{o}$ for a fixed $H_{a}=10^{-3}$. [o] solution of the steady-state Eq. (5), and [-] tendency curve from the combination of Eqs. (16), (18), and (19).

$\kappa_{\max }^{*} \approx 3.3 \times 10^{-2}$, which stays nearly constant for any $H_{a}$ and $B_{o} . \eta_{\max }^{*}$ and $\kappa_{\max }^{*}$ indicate the limits of validity of Eq. (14).

For the case of local probes, $R$ is always much smaller than $\lambda_{C}$, and as a consequence $B_{o} \ll 1$. Thence, the effect of the capillarity is negligible compared to that of the attractive term at the symmetry axis, which corresponds to say that $\eta_{0}^{*}$ is fully controlled by the London-vdW potential. Following this statement, disregarding the hydrostatic term in the steady-state Eq. (5), and analytically solving for $D^{*}$ at $r^{*}=0$, we obtain

$$
D^{*}=\eta_{0}^{*}+\sqrt{1+\left(\frac{H_{a}}{2 \kappa_{0}^{*}}\right)^{1 / 3}},
$$

which, when substituting Eq. (14), gives the dependency of $D^{*}$ on $\eta_{0}^{*}$, leading to the bifurcation diagram's construction. The resulting relationship supplies the two physically possible solutions of Eq. (5), both stable and unstable branches. Bifurcation curves obtained using Eqs. (15) and (14) are also shown in Fig. 6(a), corresponding to a fixed $B_{o}=10^{-10}$ and a range $H_{a} \in\left[10^{-8}, 10^{-1}\right]$. Furthermore, the derivative of $\eta_{0}^{*}$ with respect to $D^{*}$, as well as $\kappa_{0}^{*}$, are compared to the stable branch results in Figs. 6(b) and 6(c), respectively. In all cases, a very good accordance with the numerical solution is observed. 


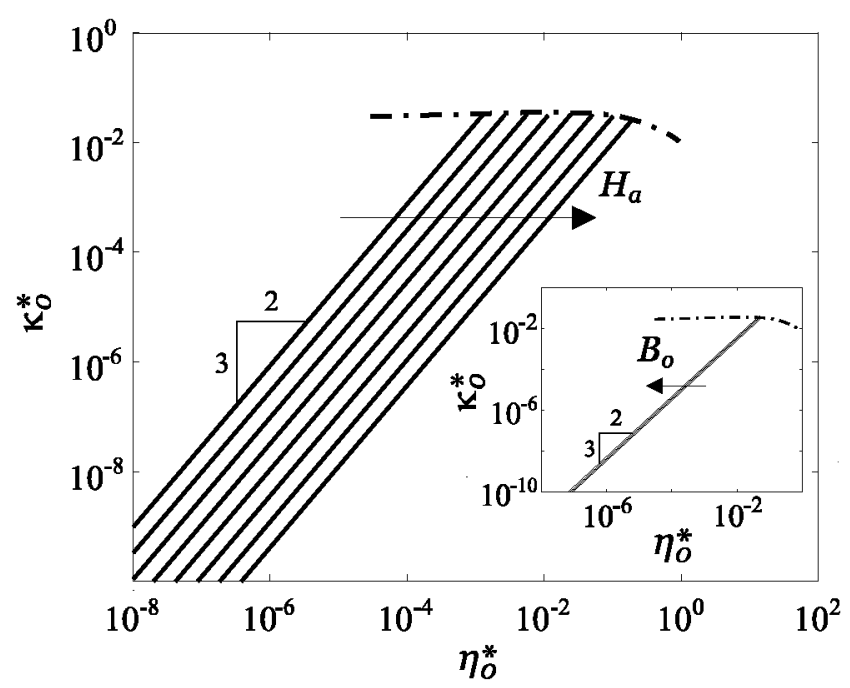

FIG. 8. Apex mean curvature $\kappa_{0}^{*}$ as a function of $\eta_{0}^{*}$ obtained for different modified Hamaker numbers in the range $H_{a} \in\left[10^{-8}, 10^{-1}\right]$ and a fixed Bond number of $B_{o}=10^{-10}$ and (inset) for different Bond numbers in the range $B_{o} \in\left[10^{-11}, 10^{-8}\right]$ and a fixed $H_{a}=10^{-3}$. [-- - ] indicates $\eta_{\max }^{*}$ obtained from the evaluation in Eq. (14) of $\eta_{\max }^{*}$, in turn obtained when solving Eq. (18). Arrows indicate the growth of the corresponding parameter.

From Fig. 1, the separation distance $D$ between the liquid surface and the probe center, and the height of the interface apex $\eta_{0}$ are related by an evident geometric relation

$$
D^{*}=1+\eta_{0}^{*}+\epsilon_{0}^{*}
$$

where $\epsilon_{0}^{*}=\epsilon_{0} / R$ is the dimensionless gap between the deformed interface and the sphere surface. Combining Eq. (16) with Eq. (15) gives the exact expression of the dimensionless gap

$$
\epsilon_{0}^{*}=\sqrt{1+\left(\frac{H_{a}}{2 \kappa_{0}^{*}}\right)^{1 / 3}}-1,
$$

which, once more with the employment of Eq. (14), also relates $\epsilon_{0}^{*}$ and $\eta_{0}^{*}$. Considering that the apex position diverges at $D_{\min }^{*}$, thus $d \eta_{0}^{*} / d D^{*} \rightarrow \infty$, we obtain $D_{\min }^{*}$ by calculating analytically the minimum of Eq. (15). Combining the latter with Eq. (14), the acquired polynomial

$$
\left(\eta_{\max }^{*}\right)^{3}+\left(\frac{H a}{2 C}\right)^{1 / 3}\left(\eta_{\max }^{*}\right)^{5 / 2}-\frac{1}{16}\left(\frac{H a}{2 C}\right)^{2 / 3}=0
$$

is meant to be solved in order to find the maximum deformation, and used to determine the minimum separation distance and the corresponding gap. The minimum gap is now given by

$$
\epsilon_{\min }^{*}=\sqrt{1+\left(\frac{H_{a}}{2 C}\right)^{1 / 3} \frac{1}{\sqrt{\eta_{\max }^{*}}}}-1,
$$

while $D_{\min }^{*}$ is obtained when substituting $\eta_{\max }^{*}$ and $\epsilon_{\min }^{*}$ in Eq. (16).

In conclusion, the employment of Eq. (14) leads to find expressions, with which we can easily determine $\eta_{\max }^{*}, \epsilon_{\min }^{*}$, and $D_{\min }^{*}$. Together with the knowledge of $H_{a}$ and $B_{o}$, which are determined using data from the literature $[1,11,12]$ or experiments [9], the optimal AFM scanning separation distance range $\left.] D_{\min }, 2 R\right]$ is found. Such information is important for imaging liquid topographies and material properties at the nanoscale.

\section{DEFORMATION SCALING}

\section{A. Characteristic length-scales}

Taking $\Pi^{*}=3 \pi R^{3} \Pi / 4 H$, we write the steady-state Eq. (5) as follows:

$$
2 \kappa^{*}+B_{o} \eta^{*}-H_{a} \Pi^{*}=0 .
$$

The absolute value of the terms appearing in Eq. (20) and the curvature decomposition terms in Eq. (9), which contribute to achieve an equilibrium steady state, are depicted in Fig. 9. The existence of three important length-scales, already introduced in Fig. 1, is emphasized.

Firstly, corresponding to the position for which $\kappa_{m}^{*}=0$ (inflection point of the meridional profile), $\lambda_{H}^{*}$ indicates the boundary of a near-field zone, and the beginning of a transition zone, where all the variables contribute to the deformation. At $r^{*} \in\left[0, \lambda_{H}^{*}\left[\right.\right.$, the attractive term $H_{a} \Pi^{*}$ (positive) is mostly opposed by $2 \kappa^{*}$ (positive), whereas the hydrostatic term $B_{o} \eta^{*}$ (positive) is negligible. Characterized by $H_{a}$, the equilibrium profile in this near-field range is directly controlled by the balance between attractive potential and capillary pressure, both showing constant magnitudes that slowly decay when $r^{*} \rightarrow \lambda_{H}^{*}$.

Then, at the radial extent for which $2 \kappa^{*}=0$ (zerocurvature), $\lambda_{T}^{*}$ marks the end of the transition region and

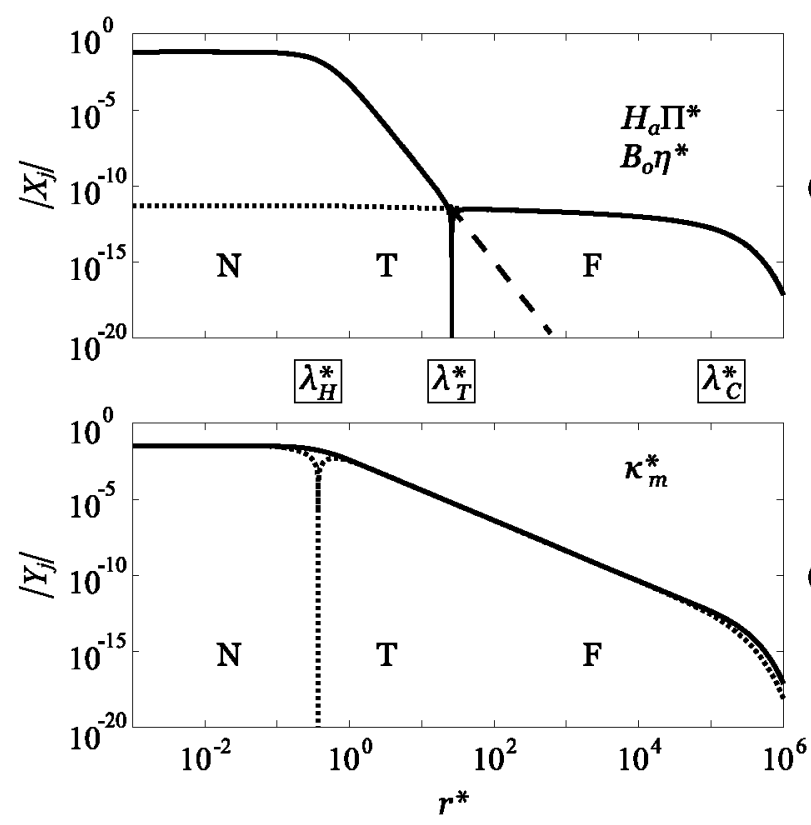

(a)

(b)

FIG. 9. Characteristic length-scales determination. Different terms (a) $X_{j}$ from Eq. (20), for which $\sum X_{j}=0$, and (b) $Y_{j}$ from Eq. (9), for which $\sum Y_{j}=2 \kappa^{*}$, as functions of $r^{*}$, for $H_{a}=10^{-3}$, $B_{o}=10^{-10}$, and $D^{*}=D_{\min }^{*}=1.168$. The uppercase letters $\mathrm{N}, \mathrm{T}$, and $F$ designate the near-field, transition, and far-field zones, respectively, which extents are bounded by the characteristic length-scales $\lambda_{H}^{*}, \lambda_{T}^{*}$, and $\lambda_{C}^{*}$. 


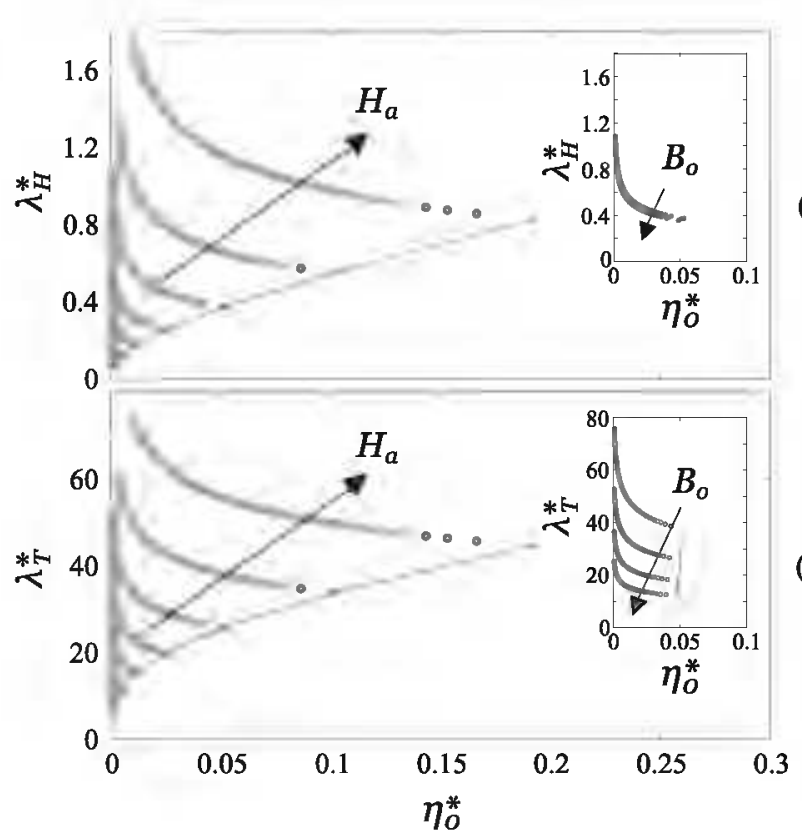

FIG. 10. (a) Radial position of the inflection point of the profile $\lambda_{H}^{*}$ and (b) radial position of zero-curvature $\lambda_{T}^{*}$, as functions of $\eta_{0}^{*}$, with the same parameter values and variables as in Fig. 8 , as well as the same considerations for the insets. Arrows indicate the growth of the corresponding parameter.

the beginning of the far-field zone, which corresponds to a capillarity dominated decay. When $\left.r^{*} \in\right] \lambda_{H}^{*}, \lambda_{T}^{*}\left[, \kappa_{a}^{*}\right.$ withstands both $H_{a} \Pi^{*}$, which quickly loses its intensity, and $\kappa_{m}^{*}$, which has adopted a negative value. In this region, $\kappa_{m}^{*}$ and $\kappa_{a}^{*}$, the latter always showing a positive value, are antagonists. $B_{o} \eta^{*}$ remains nearly constant as it slowly gains weight while both $H_{a} \Pi^{*}$ and $2 \kappa^{*}$ decrease at the same rate.

Finally, the dimensionless capillary length $\lambda_{C}^{*}$ shows the extension at which the effect of all terms tend to disappear. Within $\left.\left.r^{*} \in\right] \lambda_{T}^{*}, \lambda_{C}^{*}\right], H_{a} \Pi^{*}$ has become negligible whereas $2 \kappa^{*}$, which has become negative, attains the order of magnitude of $B_{o} \eta^{*}$. In this zone, $B_{o} \eta^{*}$, which diminishes gradually, is opposed by $2 \kappa^{*}$, while $\kappa_{m}^{*}$ and $\kappa_{a}^{*}$ are still contending. The shape of the interface profile in this far-field zone is completely given by $B_{o}$. Beyond $r^{*}=\lambda_{C}^{*}$, the interface returns to its unperturbed flat state.

In Fig. 10, $\lambda_{H}^{*}$ and $\lambda_{T}^{*}$ are shown as functions of $\eta_{0}^{*}$ for different $H_{a}$ and $B_{o}$. For a given combinations of parameters, $\lambda_{H}^{*}$ and $\lambda_{T}^{*}$ decline as functions of $\eta_{0}^{*}$, following rectangular hyperbolalike behaviors. This tendency continues until the values of $D_{\min }^{*}$ and $\eta_{\max }^{*}$ are reached, for which the limiting equilibrium profile and the minimum radial positions of $\lambda_{H}^{*}$ and $\lambda_{T}^{*}$ are attained. The numerical results indicate that $\lambda_{H}^{*}$ shifts toward higher values when $H_{a}$ increases in the range $H_{a} \in\left[10^{-8}, 10^{-1}\right]$. On the other hand, the impact of $B_{o}$ on $\lambda_{H}^{*}$, within $B_{o} \in\left[10^{-11}, 10^{-8}\right]$, is not significant. Likewise, $\lambda_{T}^{*}$ follows the same tendency when $H_{a}$ augments; however, we observe an important decrease of $\lambda_{T}^{*}$ when $B_{o}$ grows.

The curves in Fig. 10 can also be analyzed as follows. For a given $H_{a}$, a decrease of $D^{*}$ provokes the growth of

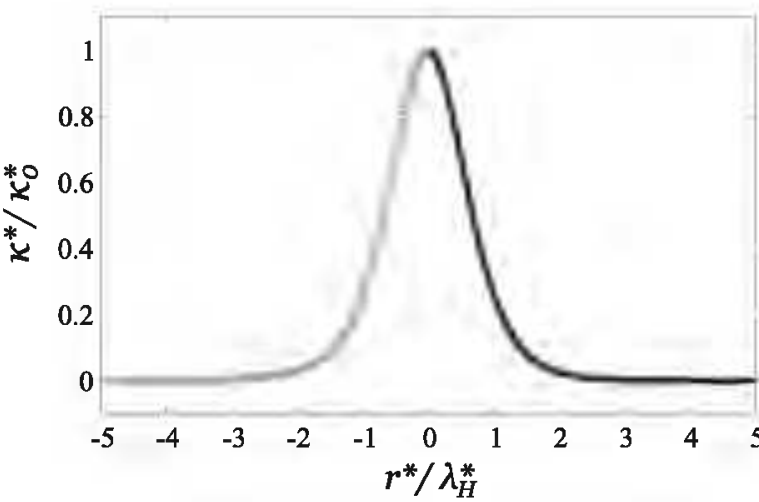

(a)

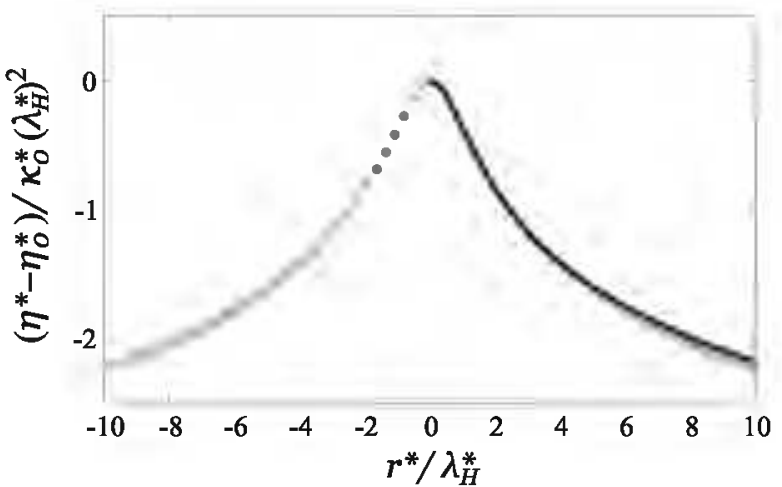

(b)

FIG. 11. Near-field self-similar dimensionless (a) curvature and (b) interface profile for $H_{a} \in\left[10^{-8}, 10^{-1}\right]$ and $B_{o} \in\left[10^{-11}, 10^{-8}\right]$. [o] numerical solution of the steady-state Eq. (5) and [-] approximations obtained from Eqs. (21) and (23), for the curvature and the interface profile, respectively.

$\Pi^{*}$ and $\eta_{0}^{*}$, leading to a decrease in both $\lambda_{H}^{*}$ and $\lambda_{T}^{*}$, which is the action of the capillary pressure to restrain the radial extent of the deformation. From another viewpoint, an increase of $H_{a}$ generates a given $\eta_{0}^{*}$ at a larger $D^{*}$, and both $\lambda_{H}^{*}$ and $\lambda_{T}^{*}$ are subsequently increased, which indicates that the attractive potential spans over a larger zone. Because $D^{*}$ and its induced $\eta_{0}^{*}$ are not significantly affected by $B_{o}$, a change in this parameter has a faint effect over $\lambda_{H}^{*}$. On the other hand, an increase of $B_{o}$ directly provokes a decrease of $\lambda_{T}^{*}$, which corresponds to enlarging the heaviness of the interface.

\section{B. Self-similarity}

Taking into account the length-scales, $\lambda_{H}^{*}$ and $\lambda_{C}^{*}$, we find master curves that display the self-similarity of the deformation profile in the near- and far-field zones, respectively. For $r^{*} \in\left[0, \lambda_{H}^{*}\left[\right.\right.$, the reduced variables $\kappa^{*} / \kappa_{0}^{*}$ and $r^{*} / \lambda_{H}^{*}$ allow us to find the dimensionless curvature presented in Fig. 11(a). This curve exhibits a logisticlike probability function shape

$$
\frac{\kappa^{*}}{\kappa_{0}^{*}}=\frac{4 \exp \left(-\frac{r^{*}}{b \lambda_{H}^{*}}\right)}{\left[1+\exp \left(-\frac{r^{*}}{b \lambda_{H}^{*}}\right)\right]^{2}},
$$

where $b$ is a fitting parameter, which is determined below. The relative error between the numerical solution of $\kappa^{*} / \kappa_{0}^{*}$ and Eq. (21) is $10^{-3}$, for a radial position up to $r^{*} \leqslant 5 \lambda_{H}^{*}$. 
In addition, the reduced curvature $\kappa^{*} / \kappa_{0}^{*}$ can be taken as negligible for radial positions beyond $r^{*} \approx 10 b \lambda_{H}^{*}$.

Matching Eq. (21) with Eq. (3), for small displacements of the liquid surface, and integrating we find

$$
\begin{aligned}
\frac{1}{r^{*}} \frac{d \eta^{*}}{d r^{*}}= & 8 \kappa_{0}^{*}\left(\frac{b \lambda_{H}^{*}}{r^{*}}\right)\left\{\frac{\exp \left(-\frac{r^{*}}{b \lambda_{H}^{*}}\right)}{1+\exp \left(-\frac{r^{*}}{b \lambda_{H}^{*}}\right)}\right. \\
& \left.+\left(\frac{b \lambda_{H}^{*}}{r^{*}}\right)\left(\ln \left[1+\exp \left(-\frac{r^{*}}{b \lambda_{H}^{*}}\right)\right]-\ln B\right)\right\},
\end{aligned}
$$

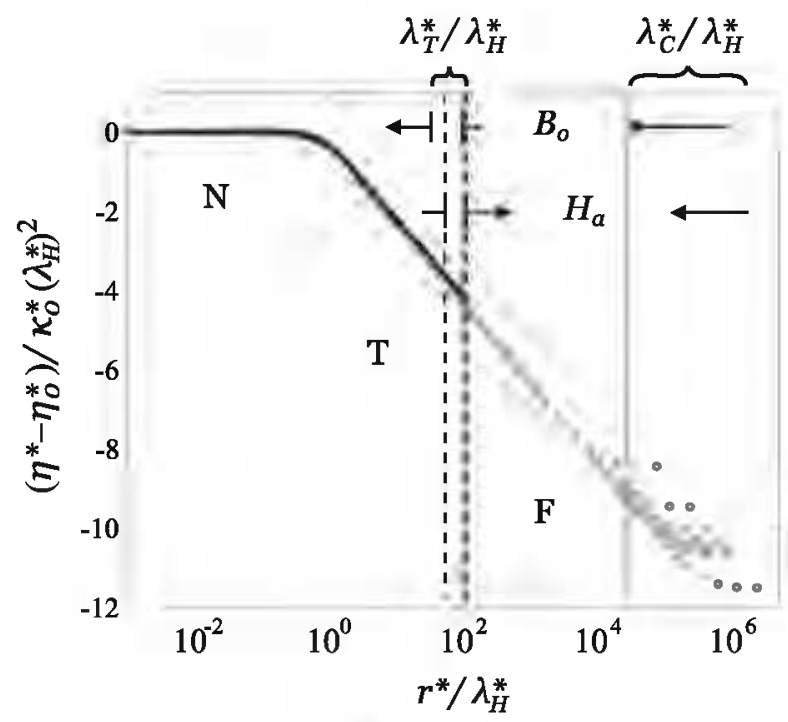

(a)

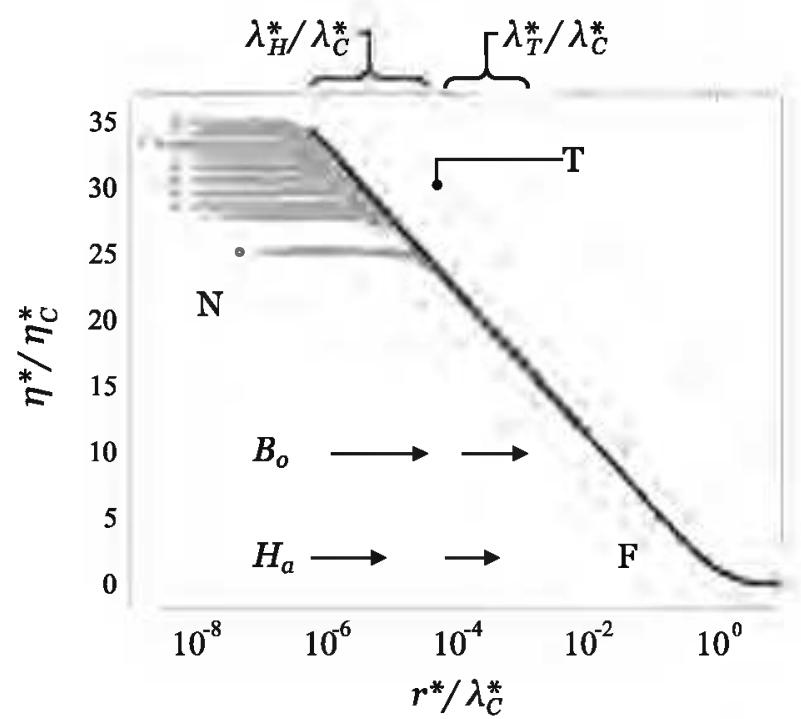

FIG. 12. Self-similar deformation profile obtained with the dimensionless variables in (a) Eq. (23) and (b) Eq. (24). [o] numerical solution of the steady-state Eq. (5) and [-] approximations obtained from Eqs. (23) and (24). The uppercase letters N, T, and F designate the near-field, transition, and far-field zones, respectively. Vertical lines indicate the positions of the reduced characteristic lengthscales, while the sense of the arrows shows its displacement when the corresponding dimensionless parameter grows: shifting $H_{a} \in$ $\left[10^{-8}, 10^{-1}\right]$ for a fixed $B_{o}=10^{-10}$, and varying $B_{o} \in\left[10^{-11}, 10^{-8}\right]$ for $H_{a}=10^{-3}$. where $B$ is an integration constant. From the boundary conditions $\left[\eta^{*}\right]_{0}^{\prime}=0$ and the corresponding Taylor expansion of the logarithmic term, we find $B=2$. As well, evaluating Eq. (22) and (21) at $r^{*}=\lambda_{H}^{*}$, where $2 \kappa^{*}=\kappa_{a}^{*}$, we deduce the value of $b=3.89 \times 10^{-1}$. Afterward, performing a second integration of $\mathrm{Eq}$. (22), we obtain the expression of the dimensionless deformation profile

$$
\begin{aligned}
\frac{\eta^{*}-\eta_{0}^{*}}{\kappa_{0}^{*}\left(\lambda_{H}^{*}\right)^{2}}= & 8 b^{2}\left\{-\ln \frac{1}{2}\left[1+\exp \left(-\frac{r^{*}}{b \lambda_{H}^{*}}\right)\right]\right. \\
& \left.+\int_{0}^{r^{*} /\left(b \lambda_{H}^{*}\right)} \ln \frac{1}{2}[1+\exp (-x)] \frac{d x}{x}\right\}
\end{aligned}
$$

where $x$ is an integration variable. Once more, the relative error between the numerical solution and $\mathrm{Eq}$. (23) is also $10^{-3}$, for a radial position up to $r^{*} \leqslant 5 \lambda_{H}^{*}$. In Fig. 11(b), the self-similar profile given by Eq. (23) is compared to the numerical results, showing a very good agreement within $0 \leqslant r^{*} \leqslant \lambda_{H}^{*}$, for $H_{a} \in$ $\left[10^{-8}, 10^{-1}\right]$ and $B_{o} \in\left[10^{-11}, 10^{-8}\right]$. Therefore, it is proven that $\left(\eta^{*}-\eta_{0}^{*}\right) / \kappa_{0}^{*}\left(\lambda_{H}^{*}\right)^{2}$ and $r^{*} / \lambda_{H}^{*}$ are the dimensionless variables that characterize the self-similar behavior of the deformation in the near-field zone. The logarithmic plot of these parameters, depicted in Fig. 12(a), exposes that this self-similarity spans largely over the transition zone.

For $\left.\left.r^{*} \in\right] \lambda_{T}^{*}, \lambda_{C}^{*}\right]$, the couple of reduced variables $\eta^{*} / \eta_{C}^{*}$ and $r^{*} / \lambda_{C}^{*}$, where $\eta_{C}^{*}=\eta^{*}\left(r^{*}=\lambda_{C}^{*}\right)$, leads us to find the far-field self-similar deformation profile

$$
\frac{\eta^{*}}{\eta_{C}^{*}}=\frac{K_{0}\left(\frac{r^{*}}{\lambda_{C}^{*}}\right)}{K_{0}(1)},
$$

As shown in Fig. 12(b), Eq. (24) gives a quite good description of the far-field results within $\lambda_{T}^{*}<r^{*} \leqslant \lambda_{C}^{*}$, and even spanning over the whole transition zone. The decaying behavior, described by the far-field self-similarity, is observed for $\left.r^{*} \in\right] \lambda_{H}^{*}, \infty[$.

\section{CONCLUDING REMARKS}

An extensive analysis of the deformation of a liquid surface, due to its interaction with a nanoprobe, was presented. The phenomenon was portrayed by a strongly nonlinear equation, and the effects of physical and geometric parameters over the system were considered. Mainly when the interaction within a nanoscale system is considered, our model generates a more accurate description of the interface deformation than previous works $[16,17]$, which employ the Derjaguin approximation. Furthermore, the application of our results is possible at any scale, considering realistic situations, due to the performed dimensionless approach.

The interface profile evolution, whether the system attains equilibrium or not, depends on the relative magnitude of $D^{*}$ with respect to the threshold distance $D_{\min }^{*}$, which in turn is given by the combination of the remaining parameters, $\boldsymbol{B}_{o}$ and $H_{a}$. Bifurcation diagrams relating $\eta_{0}^{*}$ and $D^{*}$ are established, in which a zone with nonexistent equilibrium is uncovered and explained, when $D^{*}<D_{\min }^{*}$. On the other hand, steady-state stable and unstable equilibrium profiles are obtained when $D^{*} \geqslant D_{\min }^{*}$, thus the attractive force is thwarted mostly by the capillary pressure. 
Three different length-scales were obtained from the analysis of the steady-state equilibrium profiles, all of them being function of $H_{a}$ and/or $B_{o}$. They determine the existence of: a near-field zone, $r^{*} \in\left[0, \lambda_{H}^{*}[\right.$, controlled by the attractive interaction/surface tension balance; a far-field zone, $r^{*} \in$ ]$\lambda_{T}^{*}, \lambda_{C}^{*}$, dominated by the gravity/surface tension interplay; and a transition zone, $\left.r^{*} \in\right] \lambda_{H}^{*}, \lambda_{T}^{*}[$, where all the variables take an important role. It was found that the two interior length-scales are affected by the size of the probe: $\lambda_{H} \lesssim R$ and $\lambda_{T} \gtrsim 10 R$, while the capillary length remains fixed $\lambda_{C} \sim$ $10^{-3}$. These length-scales are responsible for the liquid surface self-similar deformation profile.

[1] J. N. Israelachvili, Intermolecular and Surface Forces, 3rd ed. (Elsevier, Amsterdam, 2011).

[2] E. Pelletier, J. P. Montfort, and F. Lapique, J. Rheol. 38, 18 (1994).

[3] J. N. Sharpe, Handbook of Experimental Solid Mechanics (Springer, Berlin, 2008).

[4] A. Knoll, R. Magerle, and G. Krausch, Macromolecules 34, 4159 (2001).

[5] H. J. Butt, B. Cappella, and M. Kappl, Surf. Sci. Rep. 59, 1 (2005).

[6] T.-D. Li and E. Riedo, Phys. Rev. Lett. 100, 106102 (2008).

[7] M. Delmas, M. Monthioux, and T. Ondarcuhu, Phys. Rev. Lett. 106, 136102 (2011).

[8] B. A. Todd and S. J. Eppell, Langmuir 20, 4892 (2004).
The curvature of the stable profile is found to take a remarkable shape at its apex, $\kappa_{0}^{*} \propto\left(\eta_{0}^{*}\right)^{3 / 2}$. This fact and the problem geometry give rise to accurate simple formulas for computing the minimum separation distance $D_{\min }^{*}$, its corresponding maximum deformation $\eta_{\max }$ and minimum gap $\epsilon_{\min }^{*}$, when $H_{a}$ and $B_{o}$ are previously known $[1,11,12]$ or experimentally obtained [9]. The knowledge of $D_{\min }^{*}$ is fundamental when searching the optimal scanning noncontact AFM conditions, in order to avoid undesired capillarity effects. Nevertheless, the power-law relationship between $\kappa_{0}^{*}$ and $\eta_{0}^{*}$ remains a factual but empiric result, which is a topic for future studies.

[9] R. Ledesma-Alonso, D. Legendre, and P. Tordjeman, Phys. Rev. Lett. 108, 106104 (2012).

[10] H. C. Hamaker, Physica 4, 1058 (1937).

[11] L. Bergstrom, Adv. Colloid Interface Sci. 70, 125 (1997).

[12] J. Visser, Adv. Colloid Interface Sci. 3, 331 (1972).

[13] K. Mougin and H. Haidara, Europhys. Lett. 61, 660 (2003).

[14] L. F. Shampine, I. Gladwell, and S. Thompson, Solving ODEs with MATLAB (Cambridge University Press, Cambridge, 2003).

[15] F. M. Orr, L. E. Scriven, and A. P. Rivas, J. Fluid Mech. 67, 723 (1975).

[16] F. P.-A. Cortat and S. J. Miklavcic, Langmuir 20, 3208 (2004).

[17] Y. Z. Wang, D. Wu, X. M. Xiong, and J. X. Zhang, Langmuir 23, 12119 (2007). 